\title{
On Ruled Surfaces with Pseudo Null Base Curve in Minkowski 3-Space
}

\author{
Emilija Nešović, Ufuk Öztürk *, Esra B. Koç Öztürk and Kazım İlarslan \\ (Communicated by H. Hilmi Hacısalihoğlu)
}

\begin{abstract}
In this paper, we classify the ruled surfaces with a pseudo null base curve in Minkowski 3-space as spacelike, timelike and lightlike surfaces and obtain the corresponding striction curve and distribution parameter. In particular, we give some examples of lightlike developable surfaces with pseudo null base curve. As an application, we show that pseudo null curve and it's frame vectors generate new solutions of the Da Rios vortex filament equation.
\end{abstract}

Keywords: Ruled surface, Pseudo null curve, Minkowski space, Distribution parameter, Striction curve.

AMS Subject Classification (2010): 53C50; 53C40.

\section{Introduction}

The theory of the ruled surfaces plays an important role in Riemannian and semi-Riemannian differential geometry. The ruled surfaces also appear in many areas in computer aided geometric designs, surface approximations and tool path planning $([11,12])$. Besides, the spatial geometry of the ruled surfaces has been applied in the study of rational design problems in spatial mechanisms ([16]).

In Minkowski spaces, different properties of the ruled surfaces have been investigated (see, for example, $[1,4,6,8,9,14,17])$. The special kind of ruled surfaces in Minkowski 3-space are the null scrolls, having different physical applications, such as photon surfaces and Willmore surfaces ([2, 7]). In general relativity, lightlike hypersurfaces are of great importance, because they are models of event, Cauchy's and Kruskal's horizonts.

Da Rios vortex filament equation, obtained under localized induction approximation (LIA), represents a model for the motion of one-dimensional vortex filament in an incompressible, inviscid three-dimensional fluid. If $\alpha(s, t)$ is a non-null curve with non-null principal normal in Minkowski 3-space, then the motion satisfying the vortex filament equation generates a spacelike or a timelike Hasimoto surface in [5].

In papers dealing with the ruled surfaces in Minkowski spaces, the case when the base curve of the surface is a pseudo null curve, i.e. a spacelike curve whose principal normal vector $N(s)$ and binormal vector $B(s)$ are null vectors, is not considered. This situation motivated us to introduce the mentioned the class of surfaces in Minkowski 3-space. We obtain the striction curve and the distribution parameter of such surfaces and classify them as spacelike, timelike and lightlike. We give some examples of lightlike developable surfaces in Minkowski 3-space, such as tangent surface, principal normal surface and binormal surface over pseudo null base curve. As an application, we show that pseudo null curve and it's frame vectors $\{T, N, B\}$ generate new solutions of the Da Rios vortex filament equation.

\section{Preliminaries}

The Minkowski 3-space $\mathbb{E}_{1}^{3}$ is the Euclidean 3-space $\mathbb{E}^{3}$ provided with the standard flat metric given by

$$
g=-d x_{1}^{2}+d x_{2}^{2}+d x_{3}^{2}
$$


where $\left(x_{1}, x_{2}, x_{3}\right)$ is a rectangular coordinate system of $\mathbb{E}_{1}^{3}$. Since $g$ is an indefinite metric, recall that a vector $v \in \mathbb{E}_{1}^{3}$ can have one of three causal characters: it can be spacelike if $g(v, v)>0$ or $v=0$, timelike if $g(v, v)<0$ and null (lightlike) if $g(v, v)=0$ and $v \neq 0$. In particular, the norm (length) of a vector $v$ is given by $\|v\|=\sqrt{|g(v, v)|}$ and two vectors $v$ and $w$ are said to be orthogonal, if $g(v, w)=0$. Next, recall that an arbitrary curve $\alpha=\alpha(s)$ in $\mathbb{E}_{1}^{3}$, is said to be spacelike, timelike or null (lightlike), if all of its velocity vectors $\alpha^{\prime}(s)$ are respectively spacelike, timelike or null (lightlike) for all $s$, respectively.

In Minkowski 3-space, a spacelike curve whose principal normal vector $N$ and binormal vector $B$ are linearly independent null vectors, is called pseudo null curve. The Frenet formulae of pseudo null curve $\alpha$ have the form ([15])

$$
\left[\begin{array}{l}
T^{\prime} \\
N^{\prime} \\
B^{\prime}
\end{array}\right]=\left[\begin{array}{ccc}
0 & \kappa & 0 \\
0 & \tau & 0 \\
-\kappa & 0 & -\tau
\end{array}\right]\left[\begin{array}{l}
T \\
N \\
B
\end{array}\right],
$$

where the first curvature $\kappa(s)=0$ if $\alpha$ is pseudo null straight line or $\kappa(s)=1$ in all other cases. The second curvature $\tau(s)$ is arbitrary function of arclength parameter $s$ of $\alpha$. In particular, the following conditions are satisfied

$$
\begin{aligned}
& g(T, T)=1, g(N, N)=g(B, B)=0, \\
& g(T, N)=g(T, B)=0, g(N, B)=1 .
\end{aligned}
$$

Recall that the vector product of two vectors $u=\left(u_{1}, u_{2}, u_{3}\right)$ and $v=\left(v_{1}, v_{2}, v_{3}\right)$ in $\mathbb{E}_{1}^{3}$ is defined by

$$
u \times v=\left(u_{3} v_{2}-u_{2} v_{3}, u_{3} v_{1}-u_{1} v_{3}, u_{1} v_{2}-u_{2} v_{1}\right) .
$$

Therefore, for pseudo null curve with positively oriented frame $\{T, N, B\}$, the next relations are satisfied:

$$
T \times N=N, N \times B=T, B \times T=B .
$$

Lemma 2.1. Let $u, v$ and $w$ be the vectors in Minkowski space $\mathbb{E}_{1}^{3}$. Then:

(i) $\quad g(u \times v, w)=\operatorname{det}(u, v, w)$,

(ii) $\quad u \times(v \times w)=-g(u, w) v+g(u, v) w$

(iii) $g(u \times v, u \times v)=-g(u, u) g(v, v)+g(u, v)^{2}$.

Lemma 2.2. In Minkowski 3-space, the following properties are satisfied ([10]):

(i) two timelike vectors are never orthogonal;

(ii) two null vectors are orthogonal if and only if they are linearly dependent;

(iii) timelike vector is never orthogonal to a null vector.

Definition 2.1. A surface $S$ in a Minkowski 3-space is called a timelike (spacelike), if the induced metric on $S$ is indefinite (positive definite Riemannian) metric.

In particular, a spacelike or a timelike surface in Minkowski 3-space is also called a non-degenerate surface.

Definition 2.2. A surface $S$ in a Minkowski 3-space is called a lightlike (null, degenerate), if the induced metric on $S$ is degenerate.

In Euclidean 3-space, a surface $S$ is called developable surface, if it's Gaussian curvature $K$ is zero at each point of the surface. Namely, $S$ is developable surface if and only if the Gauss map of the surface is singular at any point of $S$. Developable surfaces in $\mathbb{E}^{3}$ (cylindrical surfaces, conical surfaces and tangent surfaces over a curve) are the ruled surfaces. Analogously, in Minkowski 3-space a surface $S$ is called developable surface, if the corresponding $\mathbb{R} P^{2}$-valued Gauss map is singular at each point ([3]). Developable surfaces, whose normal vector field is always lightlike, are called lightlike developables. Such surfaces are parts of one of the following surfaces: lightlike plane, lightcone, tangent surface over a curve lying in lightlike plane and tangent surface over a null curve ([3]).

\section{Ruled surfaces in $\mathbb{E}_{1}^{3}$}

Ruled surfaces are swept out by the motion of a straight line in $\mathbb{E}_{1}^{3}$. More formally, the image of the map $\phi: I \times \mathbb{R} \rightarrow \mathbb{E}_{1}^{3}$ defined by

$$
\phi(s, t)=\alpha(s)+t \mathbf{e}(s), \quad s \in I, \quad t \in \mathbb{R}
$$


is called a ruled surface in $\mathbb{E}_{1}^{3}$ where $\alpha: I \subset \mathbb{R} \rightarrow \mathbb{E}_{1}^{3}$ is a regular curve and $\mathbf{e}: I \subset \mathbb{R} \rightarrow \mathbb{E}_{1}^{3}$ is a vector field along $\alpha$. The curve $\alpha(s)$ and the vector field $\mathbf{e}(s)$ are respectively called the base curve (or a generating curve) and the director curve (or the director vector filed). The rulings of a ruled surface are the straight lines $t \longrightarrow \alpha(s)+t \mathbf{e}(s)$. If the tangent plane of the ruled surface is constant along a fixed ruling, the ruled surface is called the developable surface ([13]). Tangent planes of such surfaces depend on only one parameter. All other ruled surfaces are called the skew surfaces.

Definition 3.1. A ruled surface $S$ with parametrization (3.1) is said to be

$$
\begin{aligned}
\text { cylindrical, if } & \mathbf{e}(s) \times \mathbf{e}^{\prime}(s)=\overrightarrow{0}, \\
\text { non-cylindrical, if } & \mathbf{e}(s) \times \mathbf{e}^{\prime}(s) \neq \overrightarrow{0} .
\end{aligned}
$$

Definition 3.2. If there exists a common perpendicular to two preceding rulings of a skew surface, then the foot of the common perpendicular on the main ruling is called a central (striction) point. The locus of the central points is called the striction curve.

It is known that the striction curve $\bar{\alpha}$ can be written in terms of the base curve $\alpha$ as

$$
\bar{\alpha}(s)=\alpha(s)-\frac{g\left(\alpha^{\prime}(s), \mathbf{e}^{\prime}(s)\right)}{g\left(\mathbf{e}^{\prime}(s), \mathbf{e}^{\prime}(s)\right)} \mathbf{e}(s),
$$

where $g\left(\mathbf{e}^{\prime}(s), \mathbf{e}^{\prime}(s)\right) \neq 0$. Consequently, the parametrization of the ruled surface in terms of the striction line reads

$$
\phi(s, u)=\bar{\alpha}(s)+u \mathbf{e}(s), \quad s \in I, \quad u \in \mathbb{R} .
$$

The distribution parameter (Drall) of the ruled surface with parametrization (3.4) reads

$$
D(s)=\frac{g\left(\bar{\alpha}^{\prime}(s) \times \mathbf{e}(s), \mathbf{e}^{\prime}(s)\right)}{g\left(\mathbf{e}^{\prime}(s), \mathbf{e}^{\prime}(s)\right)},
$$

where $g\left(\mathbf{e}^{\prime}(s), \mathbf{e}^{\prime}(s)\right) \neq 0$.

The unit normal vector field on non-degenerate surface $\phi(s, u)$ is given by

$$
\eta(s, u)=\frac{\phi_{s} \times \phi_{u}}{\left\|\phi_{s} \times \phi_{u}\right\|} .
$$

\section{Striction curve and distribution parameter}

In this section, we give the necessary and the sufficient conditions for the ruled surfaces with pseudo null base curve to be the cylindrical surface with non-constant rulings. We also determine the striction curve and distribution parameter of the corresponding cylindrical and non-cylindrical ruled surfaces. Throughout this section, let $\mathbb{R}_{0}$ denote $\mathbb{R} \backslash\{0\}$.

Consider the ruled surface $S$ in Minkowski 3-space with parametrization

$$
\begin{aligned}
\phi: I \times \mathbb{R} & \rightarrow \mathbb{E}_{1}^{3} \\
\quad(s, t) & \rightarrow \phi(s, t)=\alpha(s)+t \mathbf{e}(s),
\end{aligned}
$$

where $\alpha(s)$ is a non-geodesic pseudo null base curve parameterized by arclength parameter $s$ and $\mathbf{e}(s)$ a nonconstant director vector. Note that there exists a nice relation between the director vector field $\mathbf{e}$ along $\alpha$ and the Frenet frame $\{T, N, B\}$ of $\alpha$. Namely, director vector e can be represented in the form

$$
\mathbf{e}(s)=a(s) T(s)+b(s) N(s)+c(s) B(s),
$$

where $a(s), b(s)$ and $c(s)$ are some scalar functions in arclength parameter $s$ of $\alpha$. By taking the derivative of the equation (4.2) with respect to $s$ and using the Frenet equations (2.1), we get

$$
\begin{gathered}
\mathbf{e}^{\prime}(s)=\left[a^{\prime}(s)-c(s)\right] T(s)+\left[a(s)+b^{\prime}(s)+b(s) \tau(s)\right] N(s) \\
+\left[c^{\prime}(s)-c(s) \tau(s)\right] B(s) .
\end{gathered}
$$


Theorem 4.1. The ruled surface with parametrization (4.1) and the rulings e given by (4.2) is cylindrical surface, if and only if it's rulings are given by:

(i) $\mathbf{e}(s)=b(s) N(s)$, where $b(s) \neq 0$ is some scalar function;

(ii) $\mathbf{e}(s)=a_{0} T(s)+b(s) N(s)$, where

$$
b(s)=e^{-\int \tau(s) d s}\left(c_{0}-a_{0} \int e^{\int \tau(s) d s} d s\right),
$$

$a_{0} \in \mathbb{R}_{0}$ and $c_{0} \in \mathbb{R}$

(iii) $\mathbf{e}(s)=a(s) T(s)+b_{0} N(s)$, where a(s) satiffies Bernoulli's differential equation

$$
a^{2}(s)-b_{0} a^{\prime}(s)+b_{0} a(s) \tau(s)=0,
$$

and $b_{0} \in \mathbb{R}_{0}$

(iv) $\mathbf{e}(s)=a(s) T(s)+b(s) N(s)$, where $a(s)$ and $b(s)$ are non-zero functions satisfying differential equation

$$
a^{2}+a b^{\prime}-b a^{\prime}+a b \tau=0
$$

(v) $\mathbf{e}(s)=a_{0} T(s)+b_{0} N(s)$, where $a_{0}, b_{0} \in \mathbb{R}_{0}$ and $\tau(s)=-a_{0} / b_{0}$;

(vi) $\mathbf{e}(s)=a(s) T(s)+b(s) N(s)+c(s) B(s)$, where $a(s), b(s), c(s)$ are some non-zero differentiable functions satisfying the system of equations:

$$
\begin{aligned}
b\left(c^{\prime}-c \tau\right)-c\left(a+b^{\prime}+b \tau\right) & =0, \\
a\left(a+b^{\prime}+b \tau\right)-b\left(a^{\prime}-c\right) & =0, \\
c\left(a^{\prime}-c\right)-a\left(c^{\prime}-c \tau\right) & =0 .
\end{aligned}
$$

Proof. Assume that the ruled surface with parametrization (4.1) is cylindrical surface. By using the relations (4.2) and (4.3) we find

$$
\begin{gathered}
\mathbf{e} \times \mathbf{e}^{\prime}=\left[b\left(c^{\prime}-c \tau\right)-c\left(a+b^{\prime}+b \tau\right)\right] T+\left[a\left(a+b^{\prime}+b \tau\right)-b\left(a^{\prime}-c\right)\right] N \\
+\left[c\left(a^{\prime}-c\right)-a\left(c^{\prime}-c \tau\right)\right] B .
\end{gathered}
$$

The condition $\mathbf{e} \times \mathbf{e}^{\prime}=\overrightarrow{0}$ implies the system of equations (4.4). If $a=b=0$, or $b=c=0$, or $a=0, b \neq 0, c \neq 0$, or $b=0, a \neq 0, c \neq 0$, the system (4.4) implies a contradiction. Now we consider the remain cases.

(i) If $a=c=0$, the system of equations (4.4) is satisfied for each $b$. Hence the ruling is given by $\mathbf{e}=b N$.

(ii) If $a=a_{0}=$ constant $\neq 0, c=0$, the system (4.4) implies the first order linear differential equation

$$
b^{\prime}+b \tau+a_{0}=0,
$$

whose general solution reads

$$
b(s)=e^{-\int \tau(s) d s}\left(c_{0}-a_{0} \int e^{\int \tau(s) d s} d s\right)
$$

where $c_{0} \in \mathbb{R}$ is the constant of integration. Thus $\mathbf{e}(s)=a_{0} T(s)+b(s) N(s)$.

(iii) If $b=b_{0}=$ constant $\neq 0, c=0$, the system (4.4) implies Bernoulli's differential equation

$$
a^{2}(s)-b_{0} a^{\prime}(s)+b_{0} a(s) \tau(s)=0,
$$

so the ruling has the form $\mathbf{e}(s)=a(s) T(s)+b_{0} N(s)$.

(iv) If $a \neq$ constant, $b \neq$ constant, $c=0$, from (4.4) we get differential equation

$$
a^{2}+a b^{\prime}-b a^{\prime}+a b \tau=0,
$$

and the ruling is given by $\mathbf{e}(s)=a(s) T(s)+b(s) N(s)$.

(v) If $a=a_{0} \in \mathbb{R}_{0}, b=b_{0} \in \mathbb{R}_{0}, c=0$, the system implies $\tau=-a_{0} / b_{0}$. Hence $\alpha$ is pseudo null helix and the ruling is given by $\mathbf{e}(s)=a_{0} T(s)+b_{0} N(s)$.

(vi) If $a \neq 0, b \neq 0, c \neq 0$ are some differential functions satisfying (4.4), the ruling has the form $\mathbf{e}(s)=$ $a(s) T(s)+b(s) N(s)+c(s) B(s)$.

In what follows we determine the striction curve $\bar{\alpha}$ and the distribution parameter $D$ of the ruled surface with the parametrization (4.1). The striction curve $\bar{\alpha}$ in terms of the base curve $\alpha$ is given by the relation (3.3). It has a geometric property that it is orthogonal to the ruling e, namely $g\left(\bar{\alpha}^{\prime}, \mathbf{e}^{\prime}\right)=0$. The relation (3.3) and Theorem 4.1 imply the next theorem. 
Theorem 4.2. Let $S$ be a cylindrical ruled surface in $\mathbb{E}_{1}^{3}$ with the parametrization (4.1). Then the following relations hold:

(i) If $\mathbf{e}(s)=b(s) N(s)$, the base curve $\alpha$ is the striction curve;

(ii) If $\mathbf{e}(s)=a_{0} T(s)+b(s) N(s)$, the base curve $\alpha$ is the striction curve;

(iii) If $\mathbf{e}(s)=a(s) T(s)+b_{0} N(s)$, the striction curve is given by

$$
\bar{\alpha}=\alpha-\frac{1}{a^{\prime}} \mathbf{e}
$$

where $a^{\prime} \neq 0$;

(iv) If $\mathbf{e}(s)=a(s) T(s)+b(s) N(s)$, the striction curve is given by

$$
\bar{\alpha}=\alpha-\frac{1}{a^{\prime}} \mathbf{e}
$$

where $a^{\prime} \neq 0$;

(v) If $\mathbf{e}(s)=a_{0} T(s)+b_{0} N(s)$, the base curve $\alpha$ is the striction curve;

(vi) If $\mathbf{e}(s)=a(s) T+b(s) N(s)+c(s) B(s)$, where $a, b$, c are some non-zero differentiable functions satisfying the system of equations (4.4) and $g\left(\mathbf{e}^{\prime}, \mathbf{e}^{\prime}\right) \neq 0$, the striction curve is given by

$$
\bar{\alpha}=\alpha-\frac{a^{\prime}-c}{\left(a^{\prime}-c\right)^{2}+2\left[c^{\prime}-c \tau\right]\left[a+b^{\prime}+b \tau\right]} \mathbf{e} .
$$

Without loss of generality, we may assume that the rulings of $S$ satisfy the condition

$$
g(\mathbf{e}(s), \mathbf{e}(s))=\text { constant },
$$

for all $s \in I$. Differentiating the relation (4.6) with respect to $s$, we obtain

$$
g\left(\mathbf{e}(s), \mathbf{e}^{\prime}(s)\right)=0,
$$

which means that $\mathbf{e}$ and $\mathbf{e}^{\prime}$ are the orthogonal vectors. Hence they can not be both timelike vectors. Also, one of them can not be timelike vector and another one null vector. In the next theorem for the striction curve $\bar{\alpha}$ of non-cylindrical ruled surface, we include the remained possibilities for $\mathbf{e}$ and $\mathbf{e}^{\prime}$.

Theorem 4.3. Let $S$ be a non-cylindrical ruled surface in $\mathbb{E}_{1}^{3}$ with the parametrization (4.1) and $\mathbf{e}^{\prime}$ is a non-null vector. Then the following relations hold:

(i) If $\mathbf{e}$ is a null vector and $\mathbf{e}^{\prime}$ is a spacelike vector, the striction curve of $S$ is given by

$$
\bar{\alpha}=\alpha-\frac{a^{\prime}-c}{\left(a^{\prime}-c\right)^{2}+2\left[c^{\prime}-c \tau\right]\left[a+b^{\prime}+b \tau\right]} \mathbf{e} .
$$

(ii) If $\mathbf{e}$ is a spacelike vector and $\mathbf{e}^{\prime}$ is a spacelike or a timelike vector, or $\mathbf{e}$ is a timelike vector and $\mathbf{e}^{\prime}$ is a spacelike vector, the striction curve of $S$ is given by (4.8).

The striction curve $\bar{\alpha}$ is related to the distribution parameter $D$ by the equation $\bar{\alpha}^{\prime} \times \mathbf{e}=D \mathbf{e}^{\prime}$. By using Theorem 4.2, we easily obtain the next theorem.

Theorem 4.4. Let $S$ be a cylindrical ruled surface in $\mathbb{E}_{1}^{3}$ with the parametrization (4.1). Then the following statements hold:

(i) If $\mathbf{e}(s)=b(s) N(s)$, then the distribution parameter

$$
D=b /\left(b^{\prime}+b \tau\right),
$$

where $b^{\prime}+b \tau \neq 0$;

(ii) If $\mathbf{e}(s)=a_{0} T(s)+b(s) N(s)$, then the distribution parameter

$$
D=b /\left(a_{0}+b^{\prime}+b \tau\right)
$$

where $a_{0}+b^{\prime}+b \tau \neq 0$;

(iii) If $\mathbf{e}(s)=a(s) T(s)+b_{0} N(s)$, then the distribution parameter $D=0$. 
(iv) If $\mathbf{e}(s)=a(s) T(s)+b(s) N(s)$, then the distribution parameter $D=0$.

(v) If $\mathbf{e}(s)=a_{0} T(s)+b_{0} N(s)$, then the distribution parameter

$$
D=b_{0} /\left(a_{0}+b_{0} \tau\right),
$$

where $a_{0}+b_{0} \tau \neq 0$;

(vi) If $\mathbf{e}(s)=a(s) T(s)+b(s) N(s)+c(s) B(s)$, where $a, b, c \neq 0$ are some differentiable functions satisfying the system of equations (4.4), then the distribution parameter $D=0$.

The next theorem and corollary can be proved by using Theorem 4.3.

Theorem 4.5. Let $S$ be a non-cylindrical ruled surface in $\mathbb{E}_{1}^{3}$ with the parametrization (4.1) and $\mathbf{e}^{\prime}$ is a non-null vector. Then the following statements hold:

(i) If $\mathbf{e}$ is a null vector satisfying and $g(\mathbf{e}, T)=0$, then the distribution parameter $D=0$;

(ii) If $\mathbf{e}$ is a null vector satisfying and $g(\mathbf{e}, T) \neq 0$, then the distribution parameter is given by

$$
D=\frac{b c^{\prime}-c b^{\prime}-a c-2 b c \tau}{\left(a^{\prime}-c\right)^{2}+2\left[c^{\prime}-c \tau\right]\left[a+b^{\prime}+b \tau\right]} ;
$$

(iii) If $\mathbf{e}$ is a spacelike vector and $\mathbf{e}^{\prime}$ is a spacelike or a timelike vector, or $\mathbf{e}$ is a timelike vector and $\mathbf{e}^{\prime}$ is a spacelike vector, then the distribution parameter is given by the relation (4.9).

Proof. (i) Assume that e is a null vector satisfying and $g(\mathbf{e}, T)=0$. By using the relation (4.2) we get $a=0$. Since $S$ is a non-cylindrical surface, the condition $g(\mathbf{e}, \mathbf{e})=0$ implies $b=0$. Hence $\mathbf{e}=c B, c \neq 0$ and therefore $\mathbf{e}^{\prime}=-c T+\left(c^{\prime}-c \tau\right) B$. By using (2.3) it follows that $\mathbf{e} \times \mathbf{e}^{\prime}=-c \mathbf{e}, c \neq 0$. Consequently, $g\left(\bar{\alpha}^{\prime}, \mathbf{e} \times \mathbf{e}^{\prime}\right)=0$, and thus $D(s)=0$.

(ii) Assume that $\mathbf{e}$ is a null vector satisfying and $g(\mathbf{e}, T) \neq 0$. Then the relation (4.2) implies $a \neq 0$ and hence $b \neq 0$ and $c \neq 0$. From (3.3) and (4.5) we get

$$
g\left(\bar{\alpha}^{\prime}, \mathbf{e} \times \mathbf{e}^{\prime}\right)=g\left(T, \mathbf{e} \times \mathbf{e}^{\prime}\right)=b c^{\prime}-c b^{\prime}-a c-2 b c \tau .
$$

By using the (3.5) and the last relation, we obtain case (ii) of the theorem.

(iii) The proof is analogous to the proof of statement (ii).

\section{The spacelike, timelike and lightlike ruled surfaces with a pseudo null base curve}

In this section, we classify the cylindrical and non-cylindrical ruled surfaces with a pseudo null base curve as spacelike, timelike and lightlike surfaces.

Let us first classify a non-cylindrical ruled surfaces with parametrization (4.1). We distinguish two cases: (I) $\mathbf{e}^{\prime}$ is a non-null vector; (II) $\mathbf{e}^{\prime}$ is a null vector.

(I) $\mathrm{e}^{\prime}$ is a non-null vector. The parametrization of $S$ in terms of the striction curve $\bar{\alpha}$ reads

$$
\phi(s, u)=\bar{\alpha}(s)+u \mathbf{e}(s), \quad s \in I, u \in \mathbb{R} .
$$

By taking the partial derivatives of the relation (5.1) with respect to $s$ and $u$ respectively, we obtain

$$
\phi_{s}=\bar{\alpha}^{\prime}(s)+u \mathbf{e}^{\prime}(s), \quad \phi_{u}=\mathbf{e}(s) .
$$

By using the last relation we find

$$
\phi_{s} \times \phi_{u}=\left(\bar{\alpha}^{\prime}(s)+u \mathbf{e}^{\prime}(s)\right) \times \mathbf{e}(s)=D(s) \mathbf{e}^{\prime}(s)+u \mathbf{e}^{\prime}(s) \times \mathbf{e}(s),
$$

where $D(s)$ is the distribution parameter of $S$.

Lemma 2.1 and the relations (4.7) and (5.2) imply

$$
g\left(\phi_{s} \times \phi_{u}, \phi_{s} \times \phi_{u}\right)=g\left(\mathbf{e}^{\prime}(s), \mathbf{e}^{\prime}(s)\right)\left[D^{2}(s)-u^{2} g(\mathbf{e}(s), \mathbf{e}(s))\right] .
$$

By using the last relation, we may determine the causal character of the normal vector field $\phi_{s} \times \phi_{u}$ on $S$, which allows us to classify the surface $S$ with parametrization (5.1) as spacelike, timelike, or lightlike surface. 
Theorem 5.1. The non-cylindrical ruled surface $S$ with the parametrization (5.1) and the non-null vector $\mathbf{e}^{\prime}$ is a spacelike surface in $\mathbb{E}_{1}^{3}$, if and only if one of the following statements hold:

(i) $\mathbf{e}$ and $\mathbf{e}^{\prime}$ are spacelike vectors, $D(s)=0$ or $D(s) \neq 0$ and $|D(s)|<|u|\|\mathbf{e}(s)\|$;

(ii) $\mathbf{e}$ is a spacelike vector, $\mathbf{e}^{\prime}$ is a timelike vector and $|D(s)|>|u||| \mathbf{e}(s)||$.

Theorem 5.2. The non-cylindrical ruled surface $S$ with the parametrization (5.1) and the non-null vector $\mathbf{e}^{\prime}$ is a timelike surface in $\mathbb{E}_{1}^{3}$, if and only if one of the following statements hold:

(i) $\mathbf{e}$ is a timelike vector and $\mathbf{e}^{\prime}$ is a spacelike vector;

(ii) $\mathbf{e}$ and $\mathbf{e}^{\prime}$ are spacelike vectors and $|D(s)|>|u|\|\mathbf{e}(s)\|$;

(iii) $\mathbf{e}$ is a null vector, $\mathbf{e}^{\prime}$ is a spacelike vector and $D(s) \neq 0$;

(iv) $\mathbf{e}$ is a spacelike vector, $\mathbf{e}^{\prime}$ is a timelike vector and $|D(s)|<|u||| \mathbf{e}(s)||$.

Theorem 5.3. The non-cylindrical ruled surface $S$ with the parametrization (5.1) and the non-null vector $\mathbf{e}^{\prime}$ is a lightlike surface in $\mathbb{E}_{1}^{3}$, if and only if $\mathbf{e}$ is a null vector, $\mathbf{e}^{\prime}$ is a spacelike vector and $D(s)=0$.

(II) $\mathbf{e}^{\prime}$ is a null vector. Then $\mathbf{e}$ is a spacelike vector. Assume that the ruled surface $S$ has the parametrization (4.1). By taking the partial derivatives of the relation (4.1) with respect to $s$ and $t$ respectively, we get

$$
\begin{aligned}
\phi_{s} & =\alpha^{\prime}+t \mathbf{e}^{\prime}=T+t \mathbf{e}^{\prime} \\
\phi_{t} & =\mathbf{e}
\end{aligned}
$$

Without loss of generality, we may assume that

$$
g(\mathbf{e}, \mathbf{e})=1 .
$$

By using the relations (5.3), (5.4) and (5.5), we obtain that the coefficients of the first fundamental form of $S$ are given by

$$
\begin{aligned}
& E=g\left(\phi_{s}, \phi_{s}\right)=1+2 t\left(a^{\prime}(s)-c(s)\right) \\
& F=g\left(\phi_{s}, \phi_{t}\right)=a(s) \\
& G=g\left(\phi_{t}, \phi_{t}\right)=1
\end{aligned}
$$

The last three relations yield

$$
g\left(\phi_{s} \times \phi_{t}, \phi_{s} \times \phi_{t}\right)=-E G+F^{2}=a^{2}-1-2 t\left(a^{\prime}-c\right) .
$$

Depending on the causal character of the normal vector field $\phi_{s} \times \phi_{t}$, we classify these surfaces in the following way.

Theorem 5.4. Let $S$ be a non-cylindrical ruled surface in $\mathbb{E}_{1}^{3}$ with the parametrization (4.1) such that $g(\mathbf{e}, \mathbf{e})=1$ and $g\left(\mathbf{e}^{\prime}, \mathbf{e}^{\prime}\right)=0$. Then:

(i) $S$ is a spacelike surface, if and only if $a^{2}(s)-1-2 t\left(a^{\prime}(s)-c(s)\right)<0$;

(ii) $S$ is a timelike surface if and only if $a^{2}(s)-1-2 t\left(a^{\prime}(s)-c(s)\right)>0$;

(iii) $S$ is a lightlike surface if and only if it has the spacelike rulings given by $\mathbf{e}(s)= \pm T(s)$, or by $\mathbf{e}(s)= \pm T(s)+$ $b(s) N(s)$, where $b^{\prime}(s)+b(s) \tau(s)+1 \neq 0$.

Corollary 5.1. Every tangent surface $\phi(s, t)=\alpha(s)+t T(s)$ over the pseudo null curve in Minkowski 3-space is the lightlike non-cylindrical ruled surface with the spacelike rulings.

The tangent surface over a curve lying in the lightlike plane in Minkowski 3-space is a lightlike developable surface ([3]). Since every pseudo null curve lies in a lightlike plane, it follows that the tangent surface over the pseudo null curve is an example of the lightlike developable surface with the spacelike rulings (Figure 1). 


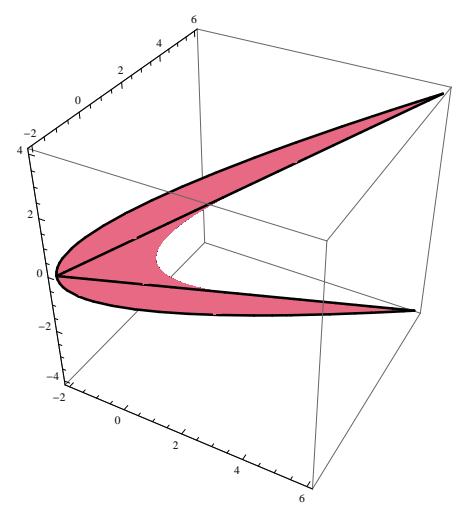

Figure 1. Tangent surface $\phi(s, t)$ over the pseudo null circle

Theorems 5.3 and 5.4 imply the next results.

Corollary 5.2. The non-cylindrical ruled surface $S$ with the pseudo null base curve is a lightlike surface in $\mathbb{E}_{1}^{3}$ if and only if it has the spacelike rulings given by $\mathbf{e}(s)= \pm T(s)$, or by $\mathbf{e}(s)= \pm T(s)+b(s) N(s)$, where $b^{\prime}(s)+b(s) \tau(s)+1 \neq 0$, or the lightlike rulings given by $\mathbf{e}(s)=c(s) B(s)$, where $c(s) \neq 0$ is some scalar function in $s$.

Corollary 5.3. Every binormal surface $\phi(s, t)=\alpha(s)+t B(s)$ over the pseudo null curve in Minkowski 3-space is a lightlike non-cylindrical ruled surface with the null rulings.

Binormal surface over a pseudo null curve is also an example of the lightlike developable surface, because it can be reparameterized as the tangent surface over a null curve, which is proved in the next theorem.

Theorem 5.5. Every binormal surface $\phi(s, t)=\alpha(s)+t B(s)$ over the pseudo null curve $\alpha$ with the torsion $\tau(s) \neq 0$ can be parameterized as the tangent surface over a null curve (Figure 2).

Proof. Let $S$ be a binormal surface over a pseudo null base curve $\alpha$ with a parametrization $\phi(s, t)=\alpha(s)+t B(s)$. Denote by $\gamma$ a null curve lying in $S$. Then $\gamma$ is given by

$$
\gamma(s)=\alpha(s)+t(s) B(s),
$$

where $s$ is arclength parameter of $\alpha$. The condition $g\left(\gamma^{\prime}(s), \gamma^{\prime}(s)\right)=0$ yields $t(s)=1$ and thus $\gamma(s)=\alpha(s)+B(s)$. Since the vector $\gamma^{\prime}(s)$ is collinear with $B(s)$, we may choose $T_{\gamma}=B(s)$. Hence by changing the directrix curve $\alpha$ to a null curve $\gamma$, we obtain the lightlike surface with the parametrization

$$
\phi(s, t)=\gamma(s)+t B(s)=\gamma(s)+t T_{\gamma}(s),
$$

which represents the tangent surface over a null curve.

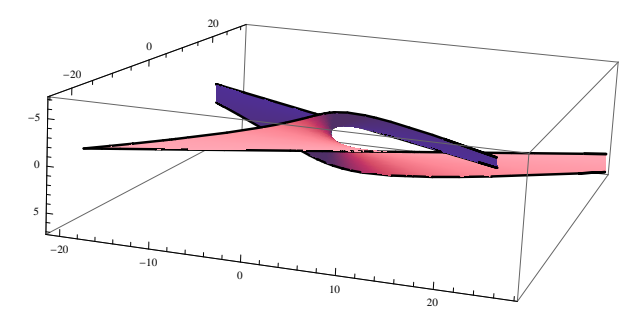

Figure 2. Binormal surface $\phi(s, t)$ over the pseudo null helix

Finally, we classify the cylindrical ruled surface $C$ with the parametrization (4.1) as a spacelike, a timelike and a lightlike surface. By using the relations (4.1) and (4.2), we find that normal vector field on $C$ is given by

$$
\phi_{s} \times \phi_{t}=T \times \mathbf{e}=b N-c B .
$$


The previous relation implies

$$
g\left(\phi_{s} \times \phi_{t}, \phi_{s} \times \phi_{t}\right)=-2 b c .
$$

Therefore, if $-2 b c>0$ the surface $C$ is timelike, and if $-2 b c<0$ the surface $C$ is spacelike. Moreover, if $b=0$, the system of equations (4.4) implies a contradition. Hence $C$ is a lightlike surface, if $c=0$.

The next two theorems can be proved by using the above relations.

Theorem 5.6. The cylindrical ruled surface $C$ in $\mathbb{E}_{1}^{3}$ with the parametrization (4.1) is a spacelike (or a timelike) surface, if and only if it has the rulings of the form $\mathbf{e}(s)=a(s) T(s)+b(s) N(s)+c(s) B(s)$, where $a, b, c \neq 0$ are some differentiable functions satisfying the system of the equations (4.4) and $\operatorname{sgn}(b)=\operatorname{sgn}(c)(\operatorname{sgn}(b) \neq \operatorname{sgn}(c))$.

Theorem 5.7. The cylindrical ruled surface $C$ in $\mathbb{E}_{1}^{3}$ with parametrization (4.1) is a lightlike surface, if and only if it's rulings are given by one of the statements (i)-(v) in Theorem 4.1.

Corollary 5.4. Every principal normal surface $\phi(s, t)=\alpha(s)+t N(s)$ over the pseudo null curve in Minkowski 3-space is a lightlike cylindrical ruled surface with the null rulings (Figure 3).

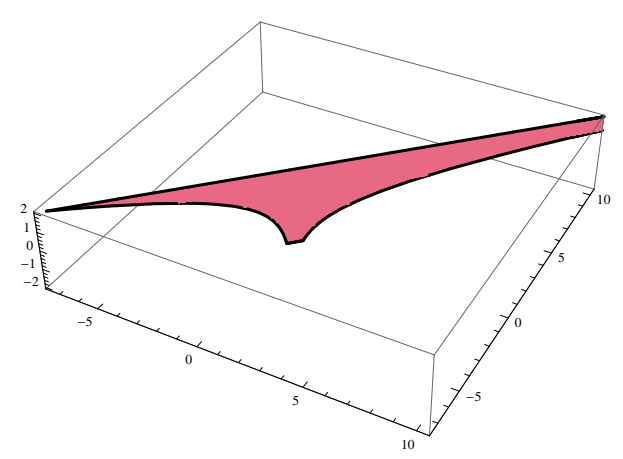

Figure 3. Principal normal surface $\phi(s, t)$ over the pseudo null helix

It is known that the only lightlike generalized cylinders in $\mathbb{E}_{1}^{3}$ are the lightlike planes ([8]), which represent lightlike developable surfaces ([3]). Hence the principal normal surface is a part of the lightlike plane and thus it is a lightlike developable surface.

\section{On solutions of Da Rios vortex filament equation}

Some classes of ruled surfaces, which are the solutions of Da Rios vortex filament equation are given in [5]. In this section, we show that the pseudo null curve and it's Frenet frame in Minkowski 3-space generate new solutions $\phi(s, t)$ of Da Rios vortex filament equation

$$
\phi_{t}=\phi_{s} \times \phi_{s s} .
$$

By taking the partial derivatives of the relation (4.1) with respect to $s$ and $t$ respectively and using (4.2), we obtain

$$
\phi_{s}=T+t \mathbf{e}^{\prime}, \quad \phi_{s s}=N+t \mathbf{e}^{\prime \prime}, \quad \phi_{t}=\mathbf{e}=a T+b N+c B .
$$

Consequently, $\phi(s, t)$ is the solution of Da Rios vortex filament equation (6.1), if

$$
\phi_{s} \times \phi_{s s}=\left(T+t \mathbf{e}^{\prime}\right) \times\left(N+t \mathbf{e}^{\prime \prime}\right)=a T+b N+c B .
$$

Applying (2.3) in the last relation, we get

$$
N+t\left(T \times \mathbf{e}^{\prime \prime}+\mathbf{e}^{\prime} \times N\right)+t^{2}\left(\mathbf{e}^{\prime} \times \mathbf{e}^{\prime \prime}\right)=a T+b N+c B .
$$

The last relation holds for each $t$, if and only if the next system of equations is satisfied

$$
a=c=0, \quad b=1, \quad T \times \mathbf{e}^{\prime \prime}+\mathbf{e}^{\prime} \times N=0, \quad \mathbf{e}^{\prime} \times \mathbf{e}^{\prime \prime}=0 .
$$

The above system of equations is satisfied if $\mathbf{e}(s)=N(s), \tau(s)=0$, or $\tau(s)=1 /(s+c), c \in \mathbb{R}$. In this way, the next theorem is proved. 
Theorem 6.1. Let $S$ be a ruled surface in $\mathbb{E}_{1}^{3}$ with the parametrization (4.1). Then $S$ is a solution of $D a$ Rios vortex filament equation (6.1), if and only if pseudo null base curve $\alpha(s)$ has the torsion $\tau(s)=0$ or $\tau(s)=1 /(s+c), c \in \mathbb{R}$ and $S$ is the principal normal surface with the parametrization

$$
\phi(s, t)=\alpha(s)+t N(s) .
$$

Hence the solution $\phi(s, t)$ is a part of the lightlike plane. Next we show that Frenet frame $\{T, N, B\}$ of a pseudo null curve can generate new solutions of Da Rios vortex filament equation. Consider the ruled surface with the parametrization

$$
x(s, t)=B(s)+t \mathbf{e}(s),
$$

where $B(s)$ is the binormal vector of the pseudo null curve and the ruling $\mathbf{e}$ is given by (4.2). By taking the partial derivatives of the relation (6.2) with respect to $s$ and $t$ respectively and using (2.1) and (4.2), we obtain

$$
\begin{aligned}
x_{s} & =-T-\tau B+t \mathbf{e}^{\prime}, \\
x_{s s} & =-N-\tau^{\prime} B+\tau T+\tau^{2} B+t \mathbf{e}^{\prime \prime}, \\
x_{t} & =\mathbf{e}=a T+b N+c B .
\end{aligned}
$$

The last relation implies

$$
\begin{array}{ll}
x_{s} \times x_{s s}=-\quad & \tau T+N-\tau^{\prime} B+t\left[-T \times \mathbf{e}^{\prime \prime}-\tau B \times \mathbf{e}^{\prime \prime}-\mathbf{e}^{\prime} \times N-\right. \\
& \left.\tau^{\prime} \mathbf{e}^{\prime} \times B+\tau \mathbf{e}^{\prime} \times T+\tau^{2} \mathbf{e}^{\prime} \times B\right]+t^{2} \mathbf{e}^{\prime} \times \mathbf{e}^{\prime \prime} .
\end{array}
$$

Therefore, $x(s, t)$ is the solution of Da Rios vortex filament equation (6.1), if

$$
\begin{aligned}
- & \tau T+N-\tau^{\prime} B+t\left[-T \times \mathbf{e}^{\prime \prime}-\tau B \times \mathbf{e}^{\prime \prime}-\mathbf{e}^{\prime} \times N-\right. \\
& \left.\tau^{\prime} \mathbf{e}^{\prime} \times B+\tau \mathbf{e}^{\prime} \times T+\tau^{2} \mathbf{e}^{\prime} \times B\right]+t^{2}\left[\mathbf{e}^{\prime} \times \mathbf{e}^{\prime \prime}\right]=a T+b N+c B .
\end{aligned}
$$

The last relation implies the system of equations

$$
\begin{aligned}
& a=-\tau, \quad b=1, \quad c=-\tau^{\prime}, \quad \mathbf{e}^{\prime} \times \mathbf{e}^{\prime \prime}=0, \\
& -T \times \mathbf{e}^{\prime \prime}-\tau B \times \mathbf{e}^{\prime \prime}-\mathbf{e}^{\prime} \times N-\tau^{\prime} \mathbf{e}^{\prime} \times B+\tau \mathbf{e}^{\prime} \times T+\tau^{2} \mathbf{e}^{\prime} \times B=0 .
\end{aligned}
$$

The above system of equations is satisfied, if

$$
\mathbf{e}(s)=-\tau(s) T(s)+N(s)-\tau^{\prime}(s) B(s),
$$

where $\tau(s)$ satisfies differential equation $\tau \tau^{\prime}-\tau^{\prime \prime}=0$. Consequently, $\tau(s)=c$, or $\tau(s)=-2 /(s+c)$, or $\tau(s)=$ $\tan \left(\frac{s}{2}+c\right), c \in \mathbb{R}$. This proves the next theorem.

Theorem 6.2. Let $\alpha$ be a pseudo null curve in $\mathbb{E}_{1}^{3}$ with the Frenet frame $\{T, N, B\}$, torsion $\tau$ and $S$ the ruled surface with the parametrization

$$
x(s, t)=B(s)+t \mathbf{e}(s) .
$$

Then $S$ is the solution of the Da Rios vortex filament equation, if and only if:

(i) $\alpha$ has the torsion $\tau(s)=c, c \in \mathbb{R}$ and $S$ is a spacelike cylindrical ruled surface with constant spacelike rulings given by (Figure 4)

$$
x(s, t)=B(s)+t[N(s)-\tau(s) T(s)] ;
$$
by

(ii) $\alpha$ has the torsion $\tau(s)=-2 /(s+c)$, and $S$ is a lightlike cylindrical ruled surface with constant null rulings given

$$
x(s, t)=B(s)+t\left[\frac{2}{s+c} T+N(s)-\frac{2}{(s+c)^{2}} B(s)\right] ;
$$

(iii) $\alpha$ has the torsion $\tau(s)=\tan \left(\frac{s}{2}+c\right), c \in \mathbb{R}$ and $S$ is a timelike cylindrical ruled surface with constant timelike rulings given by

$$
x(s, t)=B(s)+t\left[-\tan \left(\frac{s}{2}+c\right) T+N(s)-\frac{1}{2 \cos ^{2}\left(\frac{s}{2}+c\right)} B(s)\right] .
$$




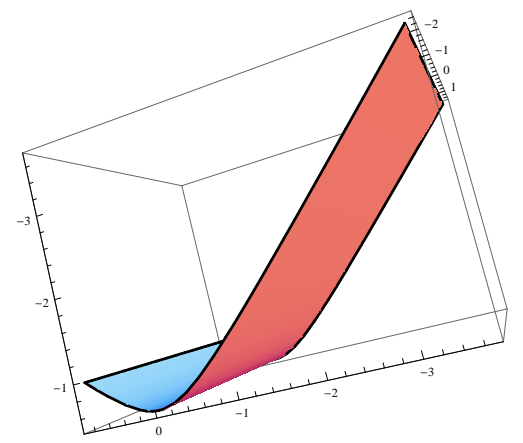

Figure 4. Cylindrical surface $x(s, t)$ over binormal $B(s)$ of pseudo null curve

\section{References}

[1] Abdel-All, N. H., Abdel-Baky, R. A., Hamdoon, F. M. Ruled surfaces with timelike rulings, Applied Mathematics and Computation, 147(2004), no. 1, 241-253.

[2] Barros, M., Ferrández, A., How big is the family of stationary null scrolls?, Journal of Geometry and Physics, 64(2013), 54-60.

[3] Chino, S., Izumiya, S., Lightlike developables in Minkowski 3-space, Demonstratio Mathematica , 43(2010), no. 2, 387-399.

[4] Dillen, F., Kuhnel, W., Ruled Weingarten surfaces in Minkowski 3-space, Manuscripta Mathematica, 98(1999), no. 3, $307-320$.

[5] Erdoğdu, M., Özdemir, M., Geometry of Hasimoto Surfaces in Minkowski 3-Space, Mathematical Physics, Analysis and Geometry, 17(2014), no. 1, 169-181.

[6] Ferrández, A., Lucas, P., On the Gauss map of B-scrolls in 3-dimensional Lorentzian space forms, Czechoslovak Mathematical Journal, 50(125) (2000), no. 4, 699-704.

[7] Foertsch, T., Hasse, W., Perlick, V., Inertial forces and photon surfaces in arbitrary spacetimes, Classical Quantum Gravity, 20(2003), no. 21, $4635-4651$.

[8] Liu, H., Ruled surfaces with lightlike ruling in Minkowski 3-space. Journal of Geometry and Physics, 59(2009), no. 1, 74-78.

[9] Liu, H., Characterizations of ruled surfaces with lightlike ruling in Minkowski 3-space, Results in Mathematics, 56(2009), no. 1-4, 357-368.

[10] O'Neill, B., Semi-Riemannian geometry with applications to relativity, Academic Press, New York, 1983.

[11] Peternell, M., Pottmann, H., Ravani, B., On the computational geometry of ruled surfaces, Computer-Aided Design, 31(1999), 17-32.

[12] Pottmann, H., Wallner, J., Approximation algorithms for developable surfaces, Comput Aided Geom Design, 16(1999), 539-556.

[13] Struik, D. J., Lectures on Classical Differential Geometry, Dover Publications, New York, 1988.

[14] Turgut, A., Hacısalihoğlu, H. H., Timelike ruled surfaces in the Minkowski 3-space. II, Turkish Journal of Mathematics, 22(1998), no. 1, 33-46.

[15] Walrave, J., Curves and surfaces in Minkowski space, Ph.D. thesis, Katholieke Universiteit Leuven, Belgium 1995.

[16] Wang, D. L., Liu, J., Xiao, D. Z., Kinematic differential geometry of a rigid body in spatial motion-II. A new adjoint approach and instantaneous properties of a line trajectory in spatial kinematics, Mechanism and Machine Theory, 32(1997), no. 4, 433-444.

[17] Yayli, Y., Saracoglu, S., On developable ruled surfaces in Minkowski space, Advances in Applied Clifford Algebras, 22(2012), no. 2, 499-510.

\section{Affiliations}

\section{E. NEŠOVIĆ}

ADDRESS: University of Kragujevac, Department of Mathematics and Informatics, 34000 Kragujevac, Serbia E-MAIL: nesovickg@sbb.rs

\section{U. ÖZTÜRK}

ADDRESS: Çankırı Karatekin University, Department of Mathematics, 18100 Çankırı, Turkey E-MAIL: ozturkufuk06@gmail.com, uuzturk@asu.edu

\section{E. B. KOÇ ÖZTÜRK}

ADDRESS: Çankırı Karatekin University, Department of Mathematics, 18100 Çankırı, Turkey

E-MAIL: e.betul.e@gmail.com, ekocoztu@asu.edu

\section{K. İLARSLAN}

ADDRESS: Kırıkkale University, Department of Mathematics, 71450 Kırıkkale, Turkey 
E-MAIL: kilarslan@yahoo.com 